\title{
Scanning capacitance microscopy characterization of AIIIBV epitaxial layers*
}

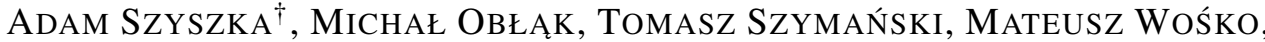 \\ WoJCIECH DAWIDOWSKI, REgINA PASZKIEWICZ
}

Wroclaw University of Technology, Faculty of Microsystem Electronics and Photonics, Janiszewskiego Street 11/17, 50-372 Wroclaw, Poland

\begin{abstract}
The applicability of scanning capacitance microscopy (SCM) technique for chosen electrical properties characterization of AIIIBV structures fabricated by Metalorganic Vapor Phase Epitaxy (MOVPE) was examined. The calibration curves for quantitative characterization of doping levels in GaAs layers were created. The AlGaN/GaN/Si heterostructures for high electron mobility transistor fabrication and InGaAs tunnel junction for tandem solar cell characterization were presented. The crucial factors of measurement conditions which could influence the obtained results were also discussed.
\end{abstract}

Keywords: scanning capacitance microscopy (SCM); atomic force microscopy (AFM); GaAs; InGaAs; AlGaN/GaN

(C) Wroclaw University of Technology.

\section{Introduction}

Continuous decrease of the characteristic dimensions of active regions of semiconductor devices is a driving force for developing advanced atomic force microscopy (AFM) techniques as scanning spreading resistance microscopy (SSRM), scanning surface potential microscopy (SSPM) and scanning capacitance microscopy (SCM) which allow us to characterize electrical parameters with nanometer spatial resolution [1-3]. In SCM, the conductive tip during imaging in contact mode is biased by alternating voltage. When the tip is scanning a semiconductor sample covered by a thin insulator layer, it forms a metal-insulator-semiconductor structure whose capacitance change induced by alternating voltage depends on free carrier concentration in the semiconductor. As a result, by measuring $\mathrm{dC} / \mathrm{dV}$ signal, it is possible to obtain information about the type and carrier concentration distribution with nanometer resolution. Although SCM method is successfully used for

\footnotetext{
${ }^{*}$ This paper was presented at the $13^{\text {th }}$ Seminar on Surfaces and Thin-Film-Structures, Szklarska Poręba, Poland, 2015.

${ }^{\dagger}$ E-mail: adam.szyszka@pwr.edu.pl
}

silicon structures qualitative and quantitative characterization $[4,5]$, its application in AIIIBV compounds investigation is restricted by the lack of reliable and well documented measurement methodology. In this paper the crucial aspects of obtaining proper SCM measurement conditions for GaAs, InGaAs and AlGaN/GaN/Si device structure characterization were discussed and the results presented.

\section{Experimental}

The measurements were performed using Veeco Multimode $\mathrm{V}$ atomic force microscope equipped with scanning capacitance microscopy mode setup. The detailed description of SCM measurement principles could be found in [6]. Here, it is worth mentioning that the measurement output - SCM signal - is defined as $\mathrm{dC} / \mathrm{dV}$ amplitude changes for the flat band condition signed by the slope of the $\mathrm{dC} / \mathrm{dV}$ curve. Three types of AIIIBV samples were measured, all grown by metalorganic vapor phase epitaxy (MOVPE): GaAs structure with the layers of different $n$ doping levels, InGaAs tunnel junction for tandem solar cells and high electron mobility transistor $\mathrm{AlGaN} / \mathrm{GaN} / \mathrm{Si}$ heterostructures. More information about the growth process parameters, 
construction and properties of each of those structures can be found in the literature [7-9]. For each type of the samples, the SCM cross section profiles and maps were obtained by scanning their surfaces cleaved and covered with a thin insulating layer of native oxide (created by their exposition to air). To make electrical contact to the samples, a GaIn eutectic mixture was used.

There are three major components which influence mostly SCM imaging: stable mechanical AFM tip-sample surface contact (I), optimized parameters of SCM measurement (II) and selection of AFM tip model (III).

(I) Setting a proper mechanical contact of AFM tip with the sample surface is necessary to obtain steady and repeatable electrical contact at different points of examined structure. This is achieved by adjusting the contact mode topography imaging parameters: AFM tip press force (too small value may result in loosing the contact, too high may lead to tip damage) as well as feedback loop parameters and scanning speed (it has to be optimized to maintain constant tip press force at different locations of the sample).

(II) The SCM mode parameters (AC voltage amplitude $\left(\mathrm{V}_{\mathrm{AC}}\right)$ and frequency, capacitance sensor frequency and $\mathrm{DC}$ bias voltage $\left(\mathrm{V}_{\mathrm{DC}}\right)$ of sample) must be adjusted to obtain the maximal value of measured signal. The sample DC bias should be chosen carefully because too high values (from our experience this is the case of $\mathrm{V}_{\mathrm{DC}}>5 \mathrm{~V}$ ) could induce some charge localized in the surface states of the semiconductor sample which, in consequence, distorts the obtained results [10]. Another source of the experimental results distortion can be introduced by stray light in the measurement chamber which, if consists of photons with a sufficiently high energy, may induce photo-carriers in the sample [11]. So, any influence of illumination (for example from laser beam in a cantilever deflection control system) on the obtained results must be also monitored.

(III) Selection of AFM tip is a crucial factor on which final quality of measurement results depends mostly. Firstly, in our research the test measurements were performed to select
AFM tip type which would be used in further experiments. Five different types being a good representation of conductive tips available currently on the market have been tested for fulfillment the following requirements: high SCM image resolution and contrast, good measurement stability and long AFM probe lifetime. The conclusions of the tests were as follows: the largest lifetime had the tip with the conductive diamond coating (DDESP-FM/NANOSensors) but, due to relatively large radius of the tip, the images resolution and contrast were poor. The best images quality was obtained using $\mathrm{Cr} / \mathrm{Pt}$ (MULTI75E-G/BudgetSensors) and $\mathrm{Pt} / \mathrm{Ir}$ (SCM-PIT/NANOSensors) coated tips but the second one guaranteed better stability and longer usage. The gold covered AFM tip (PPP-NCSTAu/NANOSensors) had a very short lifetime due to very low hardness of this metal, while the images obtained using $\mathrm{Co} / \mathrm{Cr}$ (MESP/Bruker) coated tip presented insufficient resolution. Based on these preliminary observations, all measurements, which results will be presented in the next section, were performed using $\mathrm{Pt} / \mathrm{Ir}$ coated tips with a scanning speed of $1 \mu \mathrm{m} / \mathrm{s}$ and force applied to the tip of about $200 \mathrm{nN}$ (both the values were optimized for good imaging stability with sufficient lifetime of the AFM tip).

\begin{tabular}{|l|}
\hline A: $n=2.5 \times 10^{18} \mathrm{~cm}^{-3}(250 \mathrm{~nm})$ \\
\hline$B: n=2 \times 10^{17} \mathrm{~cm}^{-3}(250 \mathrm{~nm})$ \\
\hline C: $n=3 \times 10^{16} \mathrm{~cm}^{-3}(250 \mathrm{~nm})$ \\
\hline$D: n=9.5 \times 10^{17} \mathrm{~cm}^{-3}(250 \mathrm{~nm})$ \\
\hline E: GaAs insulating substrate \\
\hline
\end{tabular}

Fig. 1. Schematic cross section of the test GaAs multilayer sample.

\section{Results}

\section{1. n-type doped GaAs multilayer}

The GaAs samples were prepared by MOVPE epitaxy and consisted of four $250 \mathrm{~nm}$ thick 


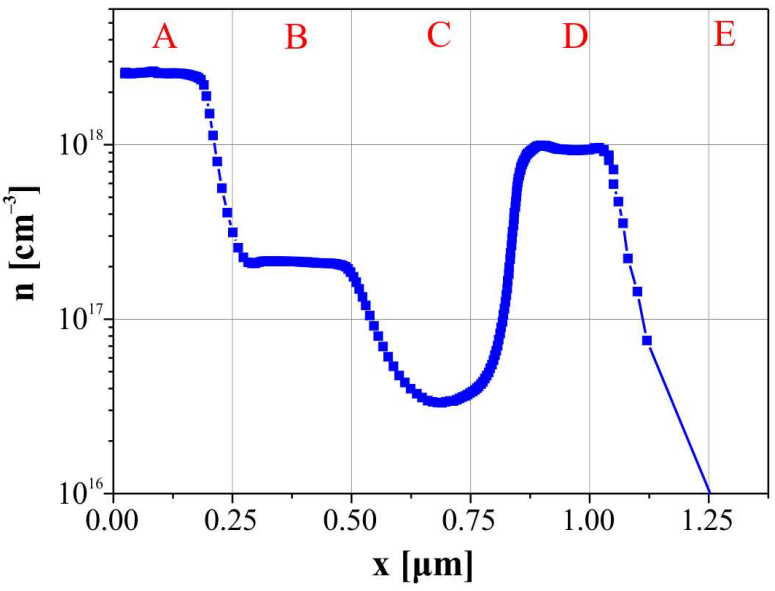

(a)

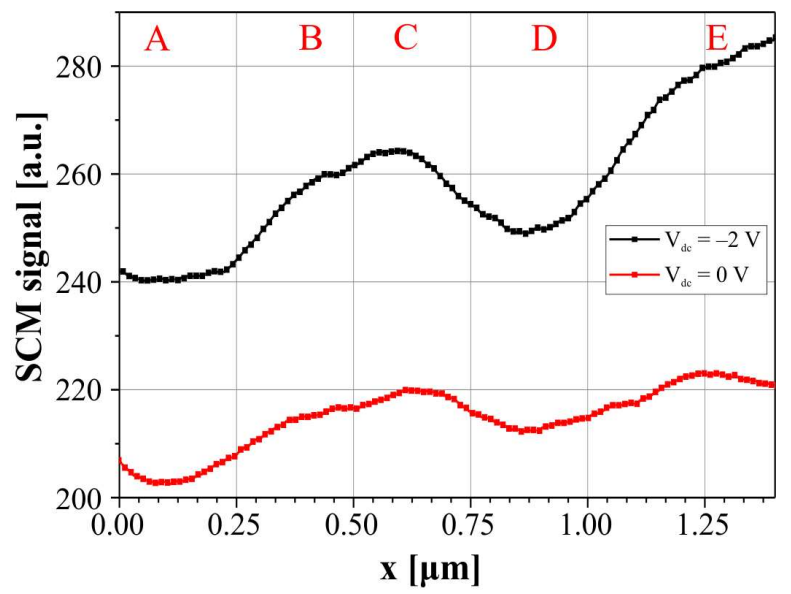

(b)

Fig. 2. E-CV (a) and SCM (b) profile of the GaAs test sample.

n-type layers with different carrier concentration (A: $\mathrm{n}=2.5 \times 10^{18} \mathrm{~cm}^{-3}$, B: $\mathrm{n}=2.0 \times 10^{17} \mathrm{~cm}^{-3}$, C: $\mathrm{n}=3.0 \times 10^{16} \mathrm{~cm}^{-3}, \mathrm{D}: \mathrm{n}=9.5 \times 10^{17} \mathrm{~cm}^{-3}$ and E: insulating substrate $\mathrm{n} \sim 1 \times 10^{16} \mathrm{~cm}^{-3}$ ) deposited on an insulating substrate (Fig. 1). Such structures were fabricated to verify whether the SCM method could be used for quantitative characterization of doping level concentration in GaAs layers by creating calibration curves allowing to correlate the obtained SCM signal with carrier concentration magnitude.

Fig. 2a presents the profile of carrier concentration in the GaAs test sample measured by electrochemical CV method (E-CV) (the respective layers are marked on the top). Relevant SCM signal profiles obtained for $\mathrm{V}_{\mathrm{AC}}=2 \mathrm{~V}$ and two different DC sample bias voltages $\left(\mathrm{V}_{\mathrm{DC}}=0 \mathrm{~V}\right.$ and $\left.2 \mathrm{~V}\right)$ are presented in Fig. 2b. The resulting calibration curves of the SCM signal in dependence on carrier density are presented in Fig. 3. It could be noticed that for both polarization voltages $\mathrm{V}_{\mathrm{DC}}$, the calibration curves have monotonic character and allow to unambiguous determining carrier concentration based on SCM measurement. This is a clear advantage of SCM technique over SSRM method where similar calibration curves determined for the same sample have large variability which excludes its application for quantitative characterization of GaAs layers [12].

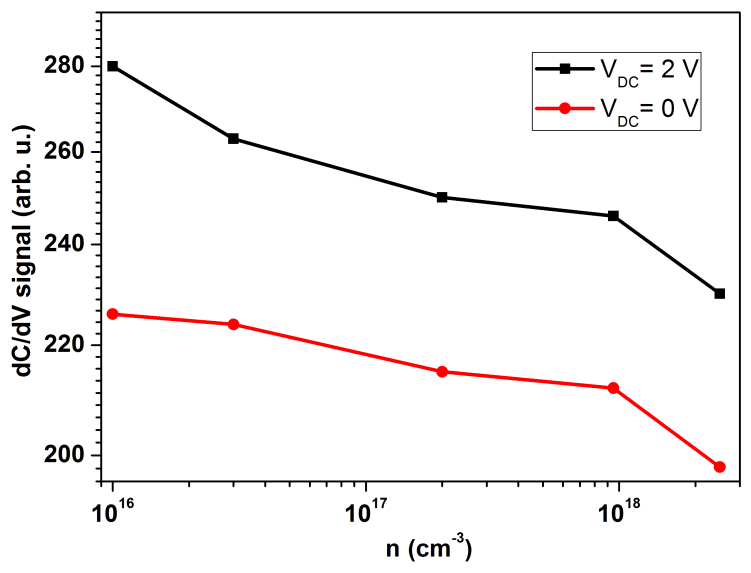

Fig. 3. Calibration curves of SCM measurement.

\subsection{InGaAs tunnel junction for tandem solar cells}

Fig. 4 presents a scheme of InGaAs tunnel junction structure intended for tandem solar cells. In the device designing process and layers growth controlling it is crucial to determine the exact position of $p-n$ electrical junction. Both E-CV and SCM methods can be used for this purpose. However, as can be seen in Fig. 5, the electrical junction position can be determined more precisely using SCM measurements. Comparison of profiles presented in the Fig. 5 shows that the electrical junction position is more precisely localized based on SCM measurement for which 
a sharp transition from negative signal ( $\mathrm{p}$ type doping) to positive (n type doping) can be easily seen. The SCM mode parameters $\left(\mathrm{V}_{\mathrm{DC}}=0.5 \mathrm{~V}\right.$ and $\mathrm{V}_{\mathrm{AC}}=1 \mathrm{~V}$ ) were selected to obtain higher difference of measured signal levels from $p$ and $n$ type doped layers. Because they differ from the parameters used during imaging of $\mathrm{n}-\mathrm{GaAs}$ test sample, the calibration curves presented in Fig. 3 could not be directly applied for quantitative characterization of this structure.

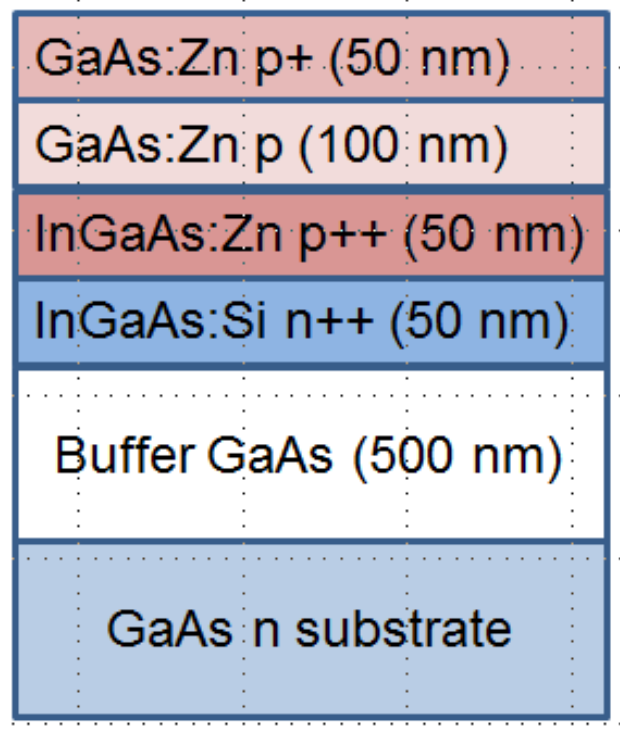

Fig. 4. Schematic cross section of InGaAs tunnel junction sample.

\subsection{AlGaN/GaN/Si heterostructures}

Schematic view of a high electron mobility transistor based on $\mathrm{AlGaN} / \mathrm{GaN}$ heterostructure grown on silicon substrate is presented in Fig. 6. Due to the difference in thermal expansion coefficient and lattice mismatch between epitaxial layer and substrate, the growth of such structure with a quality required for proper device operation is not a trivial task [13]. Basing on determined calibration curves (Fig. 3) SCM was used to verify the expected carrier concentrations levels (very high in transistor channel at $\mathrm{AlGaN} / \mathrm{GaN}$ interface and very low in the rest of the structure) across the investigated structure and also its distribution uniformity for the respective layers. Fig. 7 presents the topography profile of the sample cross section and the SCM signal profile of $\mathrm{AlGaN} / \mathrm{GaN} / \mathrm{Si}$ structure with depicted position of each layer (to prevent the AFM tip from slipping from the semiconductor layer edge, a photoresist layer was deposited on the top of the structure, which was also included in the topography). The most interesting observation is the existence of high carrier concentration not only at the $\mathrm{AlGaN} / \mathrm{GaN}$ interface but also in the region of LT (low temperature): AlN. This means that a parasitic channel for electrons exists in the structure which may result in significant deterioration of transistor parameters [14].

\section{Summary}

It was shown that SCM method can be used for semiconductor structures characterization of various AIIIBV compounds. Some factors and measurement parameters which could influence the obtained results were also described. For GaAs multilayers with different doping concentration the adequate calibration curves were obtained, which allowed quantitative carrier concentration imaging in this material. In the case of epitaxial $\mathrm{AlGaN} / \mathrm{GaN} / \mathrm{Si}$ heterostructures with a double $\mathrm{GaN}$ buffer layer, the spatial uniformity of electrical parameters was examined, in particular, the existence of a second electron conduction channel was revealed. It was also shown that in the case of InGaAs based tunnel junction, the SCM method can be successfully applied and the valuable information about the exact position of the junction can be quickly obtained.

\section{Acknowledgements}

This work was co-financed by the European Union within the European Regional Development Fund, through grant Innovative Economy (POIG.01.01.02-00-008/08-05), by the National Centre for Research and Development through the Applied Research Program Grant No. 178782, the National Centre for Research and Development through the Program LIDER, Grant No. LIDER/027/533/L533/L-5/13/NCBR/2014 and under the projects: No. PBS1/B3/2/2012 "EDEN" and No. PBS2/A3/15/2013 "PROFIT", the National Centre for Science under the Grant No. DEC-2012/07/D/ST7/02583 and by the Wroclaw University of Technology Statutory Grants and the Slovak-Polish International Cooperation Program No. SK-PL-0005-12. 


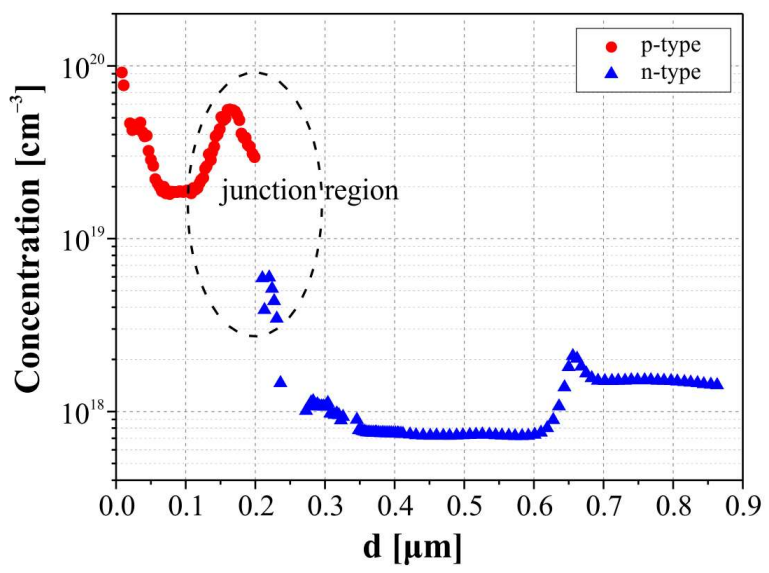

(a)

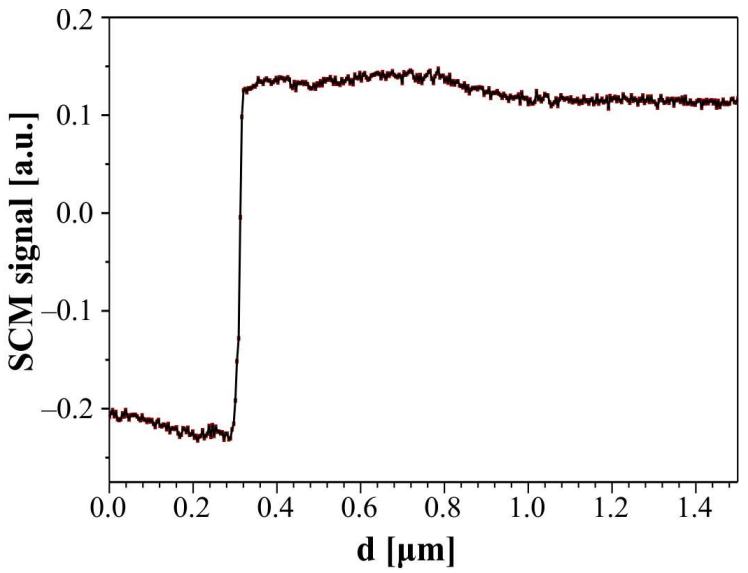

(b)

Fig. 5. E-CV (a) and SCM (b) profiles of InGaAs tunnel junction sample.

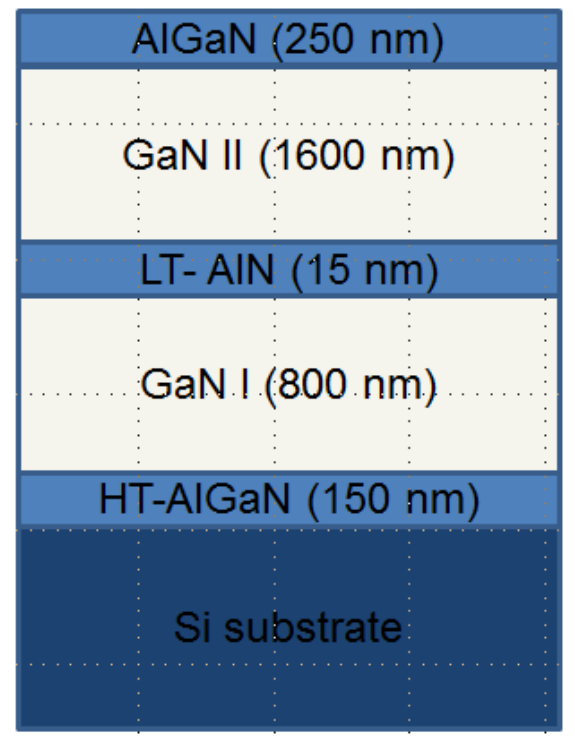

Fig. 6. Schematic cross-section of $\mathrm{AlGaN} / \mathrm{GaN} / \mathrm{Si}$ heterostructure.

\section{References}

[1] SZYSZKa A., ŚCIANA B., RADZIEWICZ D., MACHERZYŃSKI W., PASZKIEWICZ B., TŁacZaŁa M., Opt. Appl., 41 (2011), 281.

[2] Mocząa M., Sosa N., Topol A., Gotszalk T., Ultramicroscopy, 141 (2014), 1.

[3] Park K. W., Nair H. P., Crook A. M., Bank S. R., YU E. T., Appl. Phys. Lett., 99 (2011), 133114.

[4] Bassani F., Periwal P., Salem B., Chevalier N., Mariolle D., Audoit G., Gentile P., Baron T., Phys. Status Solidi-R, 8 (2014), 312.

[5] Gogheroa D., Giannazzob F., Raineria V., Mater. Sci. Eng. B-Adv., 102 (2003), 152.

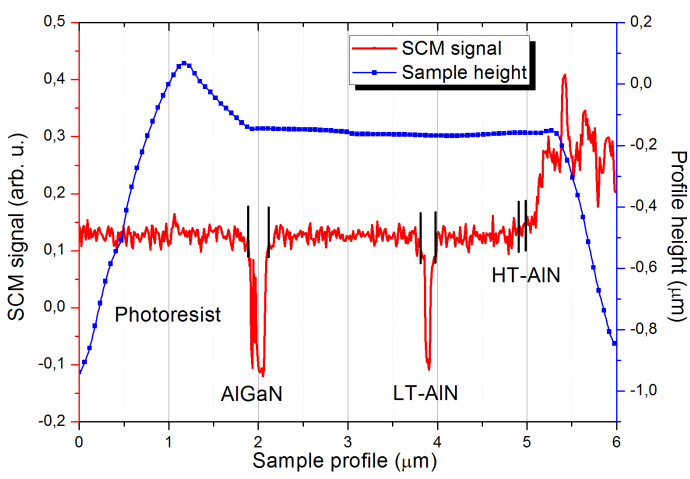

Fig. 7. Topography profile (blue) and SCM signal scan (red) of $\mathrm{AlGaN} / \mathrm{GaN} / \mathrm{Si}$ heterostructure.

[6] Bhushan B. (Ed.), Scanning Probe Microscopy in Nanoscience and Nanotechnology 2, Springer-Verlag, Berlin Heidelberg, 2001.

[7] ŚCIANA B., RADZIEWICZ D., PUCICKI D., TŁaczaŁA M., SĘK G., POlOCZEK P., Misiewicz J., KovÁČ J., SRNANEK R., Christofi A., Mater. Sci.-Poland, 26 (2008), 71.

[8] DAWIdOWSKI W., ŚCIANA B., ZBOROWSKALinder I., MiKoláŠEK M., LATKOWSKA M., RADZIEWICZ D., PUCICKI D., BIElAK K., Badura M., KováČ J., TŁaCZAŁA M., Int. J. Electron. Telecommun., 60 (2014), 151.

[9] SzYmański T., Wośko M., Paszkiewicz B., PASZKIEWICZ R., DRZIK M., J. Vac. Sci. Technol. A, 33 (2015), 041506.

[10] Smith K.V., Dang X.Z., YuA E.T., Redwing J.M., J. Vac. Sci. Technol. B, 18 (2000), 2304.

[11] YIN H., LII T., WANG W., HU W., LIN L., LU W., Appl. Phys. Lett., 95 (2009), 093506. 
[12] SzyszKa A., ŚcIana B., RADZIEWICZ MACHERZYŃSKI W., PASZKIEWICZ TŁACZAŁA M., Opt. Appl., 41 (2011), 281.

[13] Krost A., Dadgar A., Strassburger G., Clos R., Phys. Status Solidi-R, 200 (2003), 26. B.,
D., [14] Wośko M., Paszkiewicz B., Szymański T., PASzKIEwicz R., J. Cryst. Growth, 414 (2015), 248.

Received 2016-04-19 Accepted 016-09-07 